\title{
Pentaquarks in string dynamics
}

\author{
I. M. Narodetskii ${ }^{(a)}$, C. Semay ${ }^{(b)}$, B. Silvestre-Brac ${ }^{(c)}$, and Yu.A.Simonov ${ }^{(a)}$ \\ ${ }^{(a)}$ Institute of Theoretical and Experimental Physics, 117218 Moscow, Russia \\ ${ }^{(b)}$ Groupe de Physique Nucléaire Théorique, Université de Mons-Hainaut, Académie Universitaire \\ Wallonie-Bruxelles, Place du Parc 20, B-7000 Mons, Belgium \\ ${ }^{c}$ Laboratoire de Physique Subatomique et de Cosmologie, Avenue des Martyrs 53, F-38026 Grenoble-Cedex, \\ France
}

The masses of $u u d d \bar{s}$, uudd $\bar{d}$, and uuss $\bar{d}$ pentaquarks are evaluated in the framework of both the Effective Hamiltonian approach to QCD and spinless Salpeter equation using the Jaffe-Wilczek diquark approximation and the string interaction for the diquark-diquark-antiquark system. The masses of the light pentaquarks are found to be in the region above $2 \mathrm{GeV}$. Similar calculations yield the mass of $[u d]^{2} \bar{c}$ pentaquark $\sim 3250 \mathrm{MeV}$ and $[u d]^{2} \bar{b}$ pentaquark $\sim 6509$ $\mathrm{MeV}$.

\section{Introduction}

Recently LEPS 1] and DIANA 2 Collaborations reported the observation of a very narrow peak in the $K^{+} n$ and $K^{0} p$ invariant mass distribution (called $\Theta^{+}$), the existence of which has been confirmed by several experimental groups in various reaction channels. These experimental results were motivated by a pioneering paper on chiral soliton model $(\chi \mathrm{SM})$ 3. The reported mass determinations for the $\Theta^{+}$are very consistent, falling in the range $1540 \pm 10 \mathrm{MeV}$, with a width smaller than the experimental resolution of $20 \mathrm{MeV}$ for the photon and neutrino induced reactions, and of $9 \mathrm{MeV}$ for the ITEP $\mathrm{K}^{+} \mathrm{Xe} \rightarrow \mathrm{K}^{0} \mathrm{pXe}$ experiment.

In October 2003, the NA49 Collaboration at CERN SPS 44 announced evidence for an additional narrow $\Xi^{-} \pi^{-}$resonance called $\Xi^{--}(1860)$ with $I=3 / 2$, a mass $1862 \pm 2 \mathrm{MeV}$, and a width below the detector resolution of about $18 \mathrm{MeV}$. NA49 also reports evidence for a resonance $\Xi^{0}(1860)$ decaying into $\Xi^{-}(1320) \pi^{+}$. More recently, H1 collaboration at HERA [5] observed a narrow resonance $\Theta_{c}(3099)$ in $D^{*-} p$ and $D^{*+} \bar{p}$ invariant mass combinations with a mass $3099 \pm 6 \mathrm{MeV}$ and a decay width $12 \pm 3 \mathrm{MeV}$.

The complete list of both positive and negative experimental results on $\Theta^{+}(1540), \Xi^{0}(1860)$, and $\Theta_{c}(3099)$ existing by May 2004 is given, e.g., in [6]. While a dozen experiments have reported evidence for the phenomenon, another dozen haven't seen the states. In particular, the $e^{+} e^{-}$collider data (Belle, BaBar, ALEPH, DELPHI) give null results. The Fermilab high-energy proton experiments do not observe $\Xi(1860), \Theta^{+}$, and $\Theta_{c}(\mathrm{CDF})$, do not confirm $\Theta_{c}$ in both $D^{*-} p$ and $D^{-} p$ channels (FOCUS), and do not observe $\Theta \rightarrow p K_{S}^{0}$ (HyperCP, E690) [7].
Therefore, the existence of the pentaquark is, at the present time, an experimental issue. New results are expected in the near future: CLAS high statistics proton data (just done), HERMES with the double statistics (now running), COSY-TOF (five times more statistics by the end of 2004), KEK $\pi^{+} p \rightarrow \mathrm{K \Theta}^{+}$experiment E359 (will run in May $2005)$, and some others.

From the soliton point of view, the $\Theta^{+}$is not exotic as compared to other baryons - it is just a member of $\overline{\mathbf{1 0}}_{F}$ multiplet with $S=+1$. However, in the sense of the quark model, the $\Theta^{+}(1540)$ baryon with positive amount of strangeness is manifestly exotic - its minimal configuration can not be satisfied by three quarks. The positive strangeness requires a $\bar{s}$ antiquark and $q q q q$ (where $q$ refers to the lightest quarks $u, d$ ) are required for the net baryon number, thus making a pentaquark $u u d d \bar{s}$ state as the minimal "valence" configuration. Similarly, $\Theta_{c}$ can be interpreted as a pentaquark baryon with minimal quark content $(u d u d \bar{c})$; thus it is the first exotic baryon with an anti-charm quark, implying the existence of other exotic baryons with heavy quarks. The experimental evidence for the manifestly exotic baryon states provides an opportunity to refine our quantitative understanding of nonperturbative QCD at low energy.

In this talk, we explore the phenomenology of, and models for, the exotic baryons, the lowest of which is $\Theta^{+}(1540)$. The next section includes a brief review of the theoretical expectations for the masses of exotic baryons. In section 3, we provide an overview of the Effective Hamiltonian approach to QCD [8 and, in section 4, pay special attention to the dynamical calculation of pentaquark masses in the framework of this approach. Section 5 contains 
some concluding remarks.

\section{Review of Theoretical Estimations}

The $\Theta^{+}$-hyperon has hypercharge $Y=2$ and third component of isospin $I_{3}=0$. The apparent absence of $I_{3}=+1, \Theta^{++}$in the $K^{+} p$ channel argues against $I=1$; therefore it is usually assumed the $\Theta^{+}$ to be an isosinglet. The other quantum numbers are not established yet.

As to the theoretical predictions, we are faced with a somewhat ambiguous situation, in which exotic baryons may have been discovered, but there are important controversies with theoretical estimations for masses of pentaquark states. The experimental results triggered vigorous theoretical activity and put renewed urgency in the need to understand of how baryon properties are obtained from QCD.

As of today, the most successful prediction comes from the $\chi \mathrm{SM}[3$. Recently Ellis et al. 9] critically discussed the calculation of the $\Theta^{+}$and $\Xi^{--}$ masses (and widths) in the $\chi \mathrm{SM}$ and explored the unbiased theoretical and phenomenological uncertainties. Overall, they found the ranges $1432 \mathrm{MeV}<$ $m_{\Theta^{+}}<1657 \mathrm{MeV}, 1786 \mathrm{MeV}<m_{\Xi^{--}}<1970 \mathrm{MeV}$. These ranges certainly include the observed masses $m_{\Theta^{+}}=1539 \pm 2 \mathrm{MeV}$ and $m_{\Xi^{--}}=1862 \pm 2 \mathrm{MeV}$, but more precise predictions cannot be made without introducing more assumptions.

All other attempts of theoretical estimations of the pentaquark masses can be subdivided into the following five categories: i) phenomenological analyses of the $\mathrm{SU}(3)_{F}$ mass relations, ii) phenomenological analysis of the hyperfine splitting in the SakharovZeldovich (SZ) quark model in which the hadron mass is just the sum of the effective masses $m_{i}$ of its constituents plus hyperfine interaction; iii) dynamical calculations using the sum rules or lattice QCD, iv) quark model (QM) calculations, and v) dynamical calculations using the chiral SU(3) quark model.

The QCD sum rules predict a negative parity $\Theta^{+}$of mass $\simeq 1.5 \mathrm{GeV}$, while no positive parity state was found 10]. The lattice QCD calculations have claimed a pentaquark signal of either negative parity [1], [12], or positive parity [13], in the vicinity of $1540 \mathrm{MeV}$. However, the most recent quenched calculation 14 seems to reveal no evidence for a pentaquark state with quantum numbers $I\left(J^{P}\right)=0\left(\frac{1}{2}^{ \pm}\right)$ near this mass region.

The naive quark models, in which all constituents are in a relative $\mathrm{S}$-wave, naturally predict the ground state energy of a $J^{P}=\frac{1}{2}^{-}$pentaquark to be lower than that of a $J^{P}=\frac{1}{2}^{+}$one. Using the arguments based on both the Goldstone boson exchange between constituent quarks and color-magnetic exchange it was mentioned 15] that the increase of hyperfine energy in going from negative to positive parity states can be quite enough to compensate the orbital excitation energy $\sim 200-250 \mathrm{MeV}$. Existing dynamical calculations of pentaquark masses using the chiral $\mathrm{SU}(3)$ quark model (see e.g. [16]) predict the mass of the $J^{P}=\frac{1}{2}^{+}$pentaquark in the region 2 $\mathrm{GeV}$. However, the model itself is subject to significant uncertainties and the results can not be considered as conclusive.

Pentaquark baryons are unexpectedly light. Indeed, a naive QM with quark mass $\sim 350 \mathrm{MeV}$ predicts $\Theta^{+}$at about $350 \times 5=1750 \mathrm{MeV}$ plus $\sim 150 \mathrm{MeV}$ for strangeness plus $300 \mathrm{MeV}$ for the P-wave excitation. A natural remedy would be to decrease the number of constituents. This leads one to consider dynamical clustering into subsystems of diquarks like $[u d]^{2} \bar{s}$ and/or triquarks like $[u d][u d \bar{s}]$ which amplify the attractive color-magnetic forces. There are two routes that emerge naturally. One is that of [17, based on the SZ model. The other is the JaffeWilczek (JW) model [18, where it has been proposed that the systematics of exotic baryons can be explained by diquark correlations.

Neither the SZ model nor the constituent QM have been yet derived from QCD. Therefore it is tempting to consider the Effective Hamiltonian (EH) approach in QCD (see e.g. 8) which, from one side, can be derived from QCD and, from another side, leads to the results for the $\bar{q} q$ mesons and $3 q$ baryons which are equivalent to the quark model ones with some important modifications. Note that the EH approach contains the minimal number of input parameters: current (or pole) quark masses, the string tension $\sigma$, and the strong coupling constant $\alpha_{s}$, and does not contain additional fitting parameters. It should be useful and attractive to consider expanding this approach to include diquark degrees of freedom with appropriate interactions. The preview of this program based on assumption that chiral forces are responsible for the formation of $u d$ diquarks while the strings are mainly responsible for binding constituents in $\Theta^{+}$was done in [20]. In this paper we briefly review application of the $\mathrm{EH}$ approach to the JW model of pentaquarks.

In this model for the $\Theta^{+}(1540)$ and other $q^{4} \bar{q}$ baryons the four quarks are bound into two scalar, singlet isospin diquarks. Diquarks must couple to $\mathbf{3}_{c}$ to join in a color singlet hadron. In total there are six 
flavor symmetric diquark pairs $[u d]^{2},[u d][d s]_{+},[d s]^{2}$, $[d s][s u]_{+},[s u]^{2}$, and $[s u][u d]_{+}$which, combining with the remaining antiquark, give 18 pentaquark states in $\mathbf{8}_{F}$ plus $\overline{\mathbf{1 0}}_{F}$. All these states are degenerate in the $\mathrm{SU}(3)_{F}$ limit. In the $\mathrm{QM}$, the five quarks are connected by seven strings. In the diquark approximation the five-quark system effectively reduces to the three-body one, studied within the EH approach in 23 .

\section{The Effective Hamiltonian Approach}

The EH for the three constituents has the form

$H=\sum_{i=1}^{3}\left(\frac{m_{i}^{2}}{2 \mu_{i}}+\frac{\mu_{i}}{2}\right)+H_{0}+V$

where $H_{0}$ is the kinetic energy operator, $V$ is the sum of the perturbative one-gluon exchange potential and the string potential which is proportional to the total length of the strings, i.e to the sum of the distances of (anti)quark or diquarks from the string junction point. In the $\mathrm{Y}$-shape, the strings meet at $120^{\circ}$ in order to insure the minimum energy. This shape moves continuously to a two-leg configuration where the legs meet at an angle larger than $120^{\circ}$.

The constituent masses $\mu_{i}$ are expressed in terms of the current quark masses $m_{i}$ from the condition of the minimum of the hadron mass $M_{H}^{(0)}$ as a function of $\mu_{i}{ }^{1}$ :

$\frac{\partial M_{H}^{(0)}\left(m_{i}, \mu_{i}\right)}{\partial \mu_{i}}=0$

where

$M_{H}^{(0)}=\sum_{i=1}^{3}\left(\frac{m_{i}^{2}}{2 \mu_{i}}+\frac{\mu_{i}}{2}\right)+E_{0}\left(\mu_{i}\right)$.

In Eq. (3) $E_{0}\left(\mu_{i}\right)$ is an eigenvalue of the operator $H_{0}+V$. Quarks acquire constituent masses $\mu_{i} \sim$ $\sqrt{\sigma}$ due to the string interaction in (11). The $\mathrm{EH}$ in the form of (1) does not include chiral symmetry breaking effects. A possible interplay between these effects should be carefully clarified in the future.

The physical mass $M_{H}$ of a hadron is

$M_{H}=M_{H}^{(0)}+\sum_{i} C_{i}$.

1 Technically, this is done using the auxiliary field (AF) approach to get rid of the square root term in the Lagrangian 21. Applied to the QCD Lagrangian, this technique yields the EH for hadrons (mesons, baryons, pentaquarks) depending on auxiliary fields $\mu_{i}$. In practice, these fields are finally treated as $c$-numbers determined from (2).
The (negative) constants $C_{i}$ have the meaning of the constituent self energies and are explicitly expressed in terms of string tension $\sigma$ [22]

$C_{i}=-\frac{2 \sigma}{\pi \mu_{i}} \eta_{i}$

where $\eta_{q}=1, \eta_{s}=0.88$ is the correction factor due to non-vanishing current mass of the strange quark. The self-energy corrections are due to constituent spin interaction with the vacuum background fields and equal zero for any scalar constituent. The accuracy of the $\mathrm{EH}$ method for the three-quark systems is $\sim 100 \mathrm{MeV}$ 23. One can expect the same accuracy for the diquark-diquark-(anti)quark system.

Consider a pentaquark consisting of two identical diquarks with current mass $m_{[u d]}$ and an antiquark with current mass $m_{\bar{q}}(q=d, s)$. In the hyperspherical formalism the wave function $\psi(\rho, \lambda)$ expressed in terms of the Jacobi coordinates $\rho$ and $\lambda$ can be written in a symbolical shorthand as

$\psi(\rho, \lambda)=\sum_{K} \psi_{K}(R) Y_{[K]}(\Omega)$

where $R^{2}=\rho^{2}+\lambda^{2}$, and $Y_{[K]}$ are eigenfunctions (the hyperspherical harmonics) of the angular momentum operator $\hat{K}(\Omega)$ on the 6-dimensional sphere: $\hat{K}^{2}(\Omega) Y_{[K]}=-K(K+4) Y_{[K]}$, with $K$ being the grand orbital momentum. The Schrödinger equation written in terms of the variable $x=\sqrt{\mu} R$, where $\mu$ is an arbitrary scale of mass dimension which drops out in the final expressions, reads:

$$
\begin{aligned}
& \frac{d^{2} \chi_{K}(x)}{d x^{2}}+ \\
& 2\left[E_{0}+\frac{a_{K}}{x}-b_{K} x-\frac{\mathcal{L}^{2}(K)}{2 x^{2}}\right] \chi_{K}(x)=0,
\end{aligned}
$$

with the boundary condition $\chi_{K}(x) \sim \mathcal{O}\left(x^{5 / 2+K}\right)$ as $x \rightarrow 0$ and the asymptotic behavior $\chi_{K}(x) \sim$ $\operatorname{Ai}\left(\left(2 b_{K}\right)^{1 / 3} x\right)$ as $x \rightarrow \infty$. In Eq. (7) $\mathcal{L}^{2}(K)=$ $\left(K+\frac{3}{2}\right)\left(K+\frac{5}{2}\right)$,

$a_{K}=R \sqrt{\mu} \cdot \int V_{C}\left(\mathbf{r}_{1}, \mathbf{r}_{2}, \mathbf{r}_{3}\right) \cdot u_{K}^{2} \cdot d \Omega$,

where

$V_{C}\left(\mathbf{r}_{1}, \mathbf{r}_{2}, \mathbf{r}_{3}\right)=-\frac{2}{3} \alpha_{s} \cdot \sum_{i<j} \frac{1}{r_{i j}}$,

and

$b_{K}=\frac{1}{R \sqrt{\mu}} \cdot \int V_{\text {string }}\left(\mathbf{r}_{1}, \mathbf{r}_{2}, \mathbf{r}_{3}\right) \cdot u_{K}^{2} \cdot d \Omega$,

The explicit expression of $V_{\text {string }}\left(\mathbf{r}_{1}, \mathbf{r}_{2}, \mathbf{r}_{3}\right)$ in terms of Jacobi variables is given in 24]. 
For identical diquarks, like $[u d]^{2}$, the lightest state must have a wave function antisymmetric under diquark space exchange. For a state with $L=1, l_{\rho}=1, l_{\lambda}=0$ the wave function in the lowest hyperspherical approximation $K=1$ reads

$\psi=R^{-5 / 2} \chi_{1}(R) u_{1}(\Omega), u_{1}(\Omega)=\sqrt{\frac{6}{\pi^{3}}} \sin \theta \cdot\left(\mathbf{n}_{\rho}\right)_{z}$.

Here, one unit of orbital momentum between the diquarks is introduced with respect to the $\rho$ variable whereas the $\lambda$ variable is in an S-state.

In what follows, we use $\sigma=0.15 \mathrm{GeV}^{2}$, and explicitly include the Coulomb-like interactions between diquarks and antiquark with $\alpha_{s}=0.39$.

The mass of the $\Theta^{+}$obviously depends on $m_{[u d]}$ and $m_{s}$. The current masses of the light quarks are relatively well-known: $m_{u, d} \approx 0, m_{s} \approx 170 \mathrm{MeV}$. The only other parameter for strong interactions is the current mass of the diquark $m_{[u d]}$. In principle, this mass could be computed dynamically. Instead, one can tune $m_{[u d]}$ (as well as $m_{[u s]}$ and $m_{[s s]}$ ) to obtain the baryon masses in the quark-diquark approximation. We shall comment on this point later on.

\section{The results}

For pedagogy, let us first assume $m_{[u d]}=0$. This assumption leads to the lowest $u u d d \bar{d}$ and $u u d d \bar{s}$ pentaquarks. If the current diquark masses vanish, then the $[u d]^{2} \bar{d}$ pentaquark is dynamically exactly analogous to the $J^{P}=\frac{1}{2}^{-}$anti-nucleon resonance and $[u d]^{2} \bar{s}$ pentaquark is an analogue of the $J^{P}=\frac{1}{2}^{-}$ anti- $\Lambda$ hyperon, with one important exception. The masses of $\mathrm{P}$-wave baryons calculated using the $\mathrm{EH}$ method acquire the (negative) contribution $3 C_{q}$ for $N\left(\frac{1}{2}^{-}\right)$and $2 C_{q}+C_{s}$ for $\Lambda\left(\frac{1}{2}^{-}\right)$due to the constituent spin interaction with the vacuum chromomagnetic field.

However, for the scalar diquarks the above discussion shows that the self-energies $C_{[u d]}$ equal zero . This means that introducing any scalar constituent increases the pentaquark energy (relative to the $N$ and $\Lambda$ P-wave resonances by $2\left|C_{q}\right| \sim 200-300$ $\mathrm{MeV}$. Therefore prior to any calculation we can put the lower bound for the pentaquark in the JW approximation: $M(\Theta) \geq 2 \mathrm{GeV}$.

The numerical calculation for $m_{[u d]}=0$ yields the mass of the $[u d]^{2} \bar{s}$ pentaquark $\sim 2100 \mathrm{MeV}$ (see Table 1). Similar calculations yield the mass of the $[u d]^{2} \bar{c}$ pentaquark $\sim 3250 \mathrm{MeV}$ (for $m_{c}=1.4$ $\left.\mathrm{GeV}, \eta_{c}=0.234\right)$ and the mass of the $[u d]^{2} \bar{b}$ pentaquark $\sim 6509 \mathrm{MeV}$ (for $m_{b}=4.8 \mathrm{GeV}, \eta_{b}=0.052$ )
Table 1

The pentaquark masses in the diquark-diquarkantiquark approximations. Shown are the masses of $[u d]^{2} \bar{q}$ states $(q=d, s)$ for $J^{P}=\frac{1}{2}^{+}$pentaquarks.

\begin{tabular}{|c|c|c|c|c|}
\hline & & $\mu_{[u d]}$ & $\mu_{q}$ & $M$ \\
\hline \hline$[u d]^{2} \bar{s} \frac{1}{2}^{+}$ & $\mathrm{AF}$ & 0.467 & 0.470 & 2.115 \\
\cline { 2 - 5 } & $\mathrm{SSE}$ & 0.463 & 0.468 & 2.070 \\
\hline \hline$[u d]^{2} \bar{d} \frac{1}{2}^{+}$ & $\mathrm{AF}$ & 0.491 & 0.415 & 2.065 \\
\cline { 2 - 5 } & $\mathrm{SSE}$ & 0.469 & 0.379 & 1.934 \\
\hline
\end{tabular}

[26]. For illustration of the accuracy of the AF formalism, in Table1 are also shown the masses of $[u d]^{2} \bar{s}$ and $[u d]^{2} \bar{d}$ pentaquarks calculated using the spinless Salpeter equation (SSE) corresponding to the relativistic Hamiltonian :

$$
\begin{aligned}
H_{S} & =\sum_{i=1}^{3}\left(\vec{p}_{i}^{2}+m_{i}^{2}\right)^{\frac{1}{2}}+V, \\
M & =M_{0}-\frac{2 \sigma}{\pi} \sum_{i=1}^{3} \frac{\eta_{i}}{\left\langle{\sqrt{\vec{p}_{i}^{2}+m_{i}^{2}}}^{2}\right.},
\end{aligned}
$$

where $V$ is the same as in Eq. (11) ${ }^{2}$ and $M_{0}$ is the eigenvalue of $H_{S} . \mu_{[u d]}$ and $\mu_{q}$ in Table 1 denote the constituent masses calculated in the AF formalism using Eq. (5]) or the mean values $\left\langle\left(\vec{p}_{[u d]}^{2}+m_{[u d]}^{2}\right)^{\frac{1}{2}}\right\rangle$, $\left.\left\langle\left(\vec{p}_{\bar{s}}{ }^{2}+m_{\bar{s}}^{2}\right)^{\frac{1}{2}}\right)\right\rangle$ found from the solution of SSE.

Another way to estimate the current diquark masses is to tune $m_{[u d]}, m_{[u s]}$ and $m_{[s s]}$ from a fit to the nucleon and hyperon masses (in the quark-diquark approximation). We have performed such calculations using the SSE. We briefly investigated the sensitivity of the pentaquark mass predictions to the choice of the diquark masses and found that the choice $m_{[u d]}=455 \mathrm{MeV}, m_{[u s]}=565 \mathrm{MeV}$, and $m_{\bar{s}}=170 \mathrm{MeV}$ is quite acceptable. For this choice we obtain $M_{[u d] u}=1026 \mathrm{MeV}, M_{[u d] s}=1116 \mathrm{MeV}$,

${ }^{2}$ The numerical algorithm to solve the three-body SSE is based on an expansion of the wave function in terms of harmonic oscillator functions with different sizes 25. In fact to apply this techniques to the three-body SSE we need to use an approximation of the three-body potential $V_{\text {string }}$ by a sum of the twoand one-body potentials, see 27. This approximation, however, introduces a negligible correction to energy eigenvalues. 
$M_{[u s] d}=1118 \mathrm{MeV}, M_{[u s] s}=1206 \mathrm{MeV}$, yielding for the pentaquark masses

$$
\begin{aligned}
& M\left([u d]^{2} \bar{d}\right)=2377 \mathrm{MeV} \\
& M\left([u d]^{2} \bar{s}\right)=2435 \mathrm{MeV} \\
& M\left([u s]^{2} \bar{d}\right)=2533 \mathrm{MeV}
\end{aligned}
$$

These values are typically $\sim 400 \mathrm{MeV}$ higher the lowest bounds reported in Table 1 For illustration, we mention that another choice $m_{[u d]}=285 \mathrm{MeV}$, $m_{[u s]}=515 \mathrm{MeV}, m_{\bar{s}}=260 \mathrm{MeV}$ yields $M_{[u d] u}=$ $941 \mathrm{MeV}, \quad M_{[u d] s}=M_{[u s] d}=1117 \mathrm{MeV}, M_{[u s] s}=$ $1282 \mathrm{MeV}$, and the pentaquark masses

$$
\begin{aligned}
& M\left([u d]^{2} \bar{d}\right)=2288 \mathrm{MeV}, \\
& M\left([u d]^{2} \bar{s}\right)=2408 \mathrm{MeV}, \\
& M\left([u s]^{2} \bar{d}\right)=2573 \mathrm{MeV},
\end{aligned}
$$

that agree within $\sim 100 \mathrm{MeV}$ with (13).

Since two diquarks are spin and isospin singlets, there is no contribution from the spin part of one-gluon exchange potentials and spin-isospin part of Goldstone boson exchange potentials between the two diquarks and each diquark and the $\bar{s}$ quark. Only the $\sigma$ meson exchange potential produces the decrease of the pentaquark binding energy; this effect is estimated by no more than $\sim 100 \mathrm{MeV}$. The instanton induced spin interaction also produces a marginal attraction [28]. We therefore conclude that the string dynamics alone in its simplified form predicts too high masses for pentaquarks.

\section{Conclusions}

The anomalous lightness of the $\Theta^{+}$seems to unambiguously indicate a large role of chiral symmetry breaking effects in pentaquarks. The $\mathrm{QM}$ and $\chi \mathrm{SM}$ are, to a large extent, complementary. Each of these reproduces certain aspects of hadronic physics and incorporates some features of QCD that are missing in the other. An approach of $\chi \mathrm{SM}$ totally neglects the confinement effects and concentrates on the pure chiral properties of baryons. The string dynamics alone, in its simplified form, seems to predict too high masses for pentaquarks. Therefore the existence of $\Theta^{+}$, if confirmed, provides a unique possibility to clarify the interplay between the gluon and chiral degrees of freedom in light baryons.

\section{Acknowledgement}

This work was supported by RFBR grants 03-0217345, 04-02-17263, 04-02-26642 and the grant for leading scientific schools 1774.2003.2.

\section{REFERENCES}

1. T. Nakano et al., LEPS Collaboration, Phys. Rev. Lett. 91, (2003) 012002.

2. V. V. Barmin et al., DIANA Collaboration, Phys. At. Nucl. 66, (2003) 1715.

3. D. Diakonov, V. Petrov and M. V. Polyakov, Z. Phys. A 359, (1997) 305.

4. C. Alt et al., NA49 collaboration, Phys. Rev. Lett. 92, (2004) 042003

5. A. Aktas et al, H1 Collaboration, Phys. Lett. B $588,(2004) 17$

6. M. Kubantsev, talk at the International Workshop HSQCD, Repino, St. Petersburg, May 2004

7. D. Litvintsev, these proceedings.

8. Yu.A. Simonov, arXiv: hep-ph/9911237

9. J. Ellis, M. Karliner and M. Praszalowicz, arXiv: hep-ph/0401127

10. R. Matheus et al., arXiv: hep-ph/0309001. J. Sugiyama, T. Doi, M. Oka, arXiv: hep-ph/0309271

11. F. Csikor et al., JHEP 0311, (2003) 070.

12. S. Sasaki, arXiv: hep-lat/0310014.

13. T.W. Chiu, T.H. Hsieh, arXiv: hep-ph/0403020 hep-ph/0404007

14. N. Mathur et al., arXiv: hep-ph/0406196

15. Fl. Stancu and D.O. Riska, Phys.Lett. B 575, (2003) 242.

16. F. Huang et al., Phys.Lett. B586, (2004) 69; Fl. Stancu, Phys.Lett. B 595, (2004) 269.

17. M. Karliner and H.J. Lipkin, Phys. Lett. B 575, (2003) 249.

18. R.L. Jaffe, F. Wilczek, Phys. Rev. Lett. 91, (2003) 232003.

19. For a review see e.g. M. Anselmino et al., Rev. Mod. Phys. 65, (1993) 1199.

20. I.M. Narodetskii et al., Phys. Lett. B 578, (2004) 318.

21. C. Semay, B. Silvestre-Brac, I. M. Narodetskii, Phys.Rev. D69, (2004) 014003.

22. Yu.A. Simonov, Phys. Lett. B 515, (2001) 137.

23. Yu.A. Simonov, Phys. Atom. Nucl. 66, (2003) 338; I.M. Narodetskii, M.A. Trusov, Phys. Atom. Nucl. 67, (2004) 762.

24. I.M. Narodetskii, A.N. Plekhanov, and A.I. Veselov, JETP Lett. 77, (2003) 58.

25. P. Nunberg, D. Prosperi, and E. Pace, Nucl. Phys. A 285, (1977) 58.

26. A.I. Veselov, private communication.

27. B. Silvestre-Brac et al., Eur. Phys. J. C32, (2003) 385.

28. C. Semay and B. Silvestre-Brac, to appear in Eur. Phys. J. 